\title{
Does folding determine protein configuration?
}

\author{
An ingenious account of the protein-folding problem turns the usual formulation on its head, yielding the suggestion \\ that foldability is an evolutionary necessity.
}

THE problem of protein folding is notoriously one of the most difficult in biology. The starting point is the specification of the amino acids along the protein chain. There may be some hundreds of them altogether. In principle, each residue can interact with all others, so that there is a multitude of possible interactions. And many of the configurations implied by these random interactions are energetically metastable in the sense that their free energy is less than that of all configurations obtained from them by making all possible small displacements. But the free energy may nevertheless be far above the global minimum, if only that could be found. So the essence of the protein-folding problem is the question of how, in real life, a protein molecule of specified amino-acid sequence ends up in a unique configuration which, among other things, determines its biological function.

That is merely a statement of the general problem. In practice, there are other difficulties. As yet, for example, there is no systematic way of including the interaction of water molecules in the specification of the problem, but every reason to suppose that water interactions are of the essence in the function of real-life protein molecules. A further and related difficulty is that there is little hope of tackling the folding problem except at the level of atoms, of which there will be several hundreds, perhaps thousands, in a real protein. Given that, it is remarkable that people have done as well as they have in the past few years in bringing computation to bear on the problem.

Molecular dynamics has done most to make the problem approachable. The first need is for a force-field, or a specification of the interaction energy as a function of distance between all possible pairs of interacting atoms. (The peculiar difficulty arising with hydrogen bonds is that the force will depend on at least two distances, those between the proton and the two heavier atoms it is supposed to hold together.) Ideally, modellers should also allow the transfer of charge from any part of the whole molecule to any other.

In the world that lies a little further from the ideal, it would be good to tackle the problem from first principles, by calculating the geometrical configuration of the whole molecule and the structure of its valence electrons simultaneously, but a glance at any issue of the Journal of the American Chemical Society will show that the theoretical chemists are still struggling with compounds comparable with a single amino acid in complexity.

Given a usable force-field, the problem can be handed over to a machine and to one of the intricate suites of software developed for the purpose. Such a program inevitably finds metastable states as well as that whose energy is absolutely the smallest. There are built-in techniques for testing whether a particular state is that of the smallest absolute energy. One is to give the molecule as a whole an abnormally high temperature to see whether the metastable configuration then migrates to one with less energy. 'Annealing' is the vivid term for that.

But a computer simulation is rarely a solution of a real physical problem. And the fundamental difficulty with the simulation of protein configurations is that, so long as the chosen force-field is necessarily a crude approximation to the truth, no amount of precision in the computation can give a person confidence that the state with the lowest energy is really the global minimum, let alone that it approximates to that of the native protein with any accuracy.

Given all this complexity (in the strict sense), it is not surprising that many biologists declare impatiently that nature cannot possibly afford the luxury of allowing each polypeptide made by the transcription of an RNA molecule from a ribosome to go through the processes of molecular dynamics until it reaches the configuration in which it is biologically active. Indeed, there is not time for that, as the late Cyrus Leventhal pointed out in the $1960 \mathrm{~s}$. So may it be that polypeptides begin folding once they have begun to be assembled at the ribosome, so that the final state is conditioned by the first part of the protein molecule to be constructed? Alternatively, people argue, there may be a protein (call it 'templin') whose function is to be a template that determines the folding pattern of a whole class of related proteins.

E.I. Shakhnovich, a Russian at the Chemistry Department at Harvard, is now advocating a scheme for similarly finessing the difficulties of the molecular dynamics, and which, when tested, may have interesting implications for the evolution of protein molecules (Phys. Rev. Lett. 24, 3907-3910; 1994). In essence, he turns the problem of protein folding inside out. First, he says, choose a configuration for a notional protein molecule with a predetermined length and then ring all the possible changes on the amino-acid sequence until the energy of the structure is the smallest.

To make the job manageable, he has worked with a notional polypeptide of 80 amino acids and with an arbitrarily chosen configuration in which neighbouring amino acids are on neighbouring vertices of a cubic $4 \times 4 \times 5$ lattice (so that every vertex is occupied). The force-field is similarly skeletal; amino acids interact with each other only when they are on neighbouring lattice points, when their mutual energy is simply a number determined by the character of the pair concerned.

It is then possible to use conventional molecular dynamics (in this case, a simple Monte Carlo variation of the configuration is sufficient) to follow the folding of the energetically optimum polypeptide. The outcome is dramatic. The notional polypeptide hunts about for a stable conformation for 6 million time-steps in the Monte Carlo simulation, without encountering metastable states en route, until it finally clicks into the form of the notionally native protein - the tracing of a closed graph on the 80-vertex cubic lattice that Shakhnovich had thought of in the first place.

The importance of this result is not what it says about the particular (and unreal) configuration described, but that it suggests that, among all possible configurations for a protein of specified length and sequence, there may be one that allows for unambiguous and comparatively rapid folding into the native form. And once that state has been attained, thermal fluctuations do not seriously disturb the configuration: Shakhnovich estimates that his 80-residue polypeptide is folded properly 95 per cent of the time, and unfolded for 5 per cent of it. He also says that there are ample unpublished calculations to support these conclusions.

The novelty of the concept is that the protein configurations with which natural selection has endowed us may have been chosen not only for their biological activity but also for their capacity to fold unambiguously and in reasonably short order. (A protein that failed to fold would presumably be discarded quickly as a drag on fitness.) It will be particularly interesting to see whether this idea can be used to tell which amino acids at which sites in real proteins are likely to have been conserved in evolution. It will also be interesting to see the inevitable application of this line of argument to the secondary and tertiary structure of RNA molecules, where the same considerations arise. John Maddox 